\title{
ESTRUCTURA DE LOS TWEETS POLÍTICOS DURANTE LAS CAMPAÑAS ELECTORALES DE 2015 Y 2016 EN ESPAÑA
}

\section{Structure of the political tweets during the electoral campaigns of 2015 and 2016 in Spain}

Frederic Guerrero-Solé y Lluís Mas-Manchón
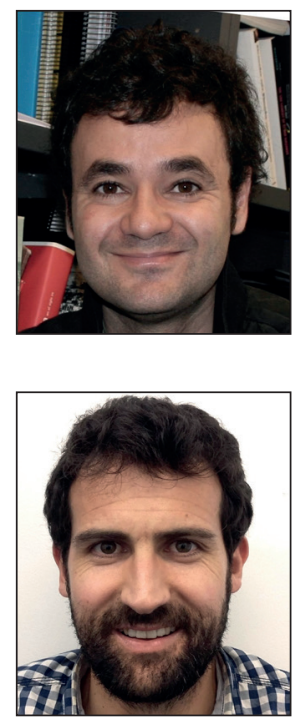

Frederic Guerrero-Solé es licenciado en Física y doctor en Comunicación por la Universidad Pompeu Fabra, en cuyo Departamento de Comunicación ejerce como profesor lector. Sus principales líneas de investigación son las redes sociales, los efectos de los medios y la sociología de la comunicación. Ha publicado diversos artículos sobre Twitter en revistas como Information, communication and society y Social science computer review.

http://orcid.org/0000-0001-8145-8707

frederic.guerrero@upf.edu

\begin{abstract}
Lluís Mas-Manchón es doctor en Comunicación Audiovisual y Publicidad por la Universidad Autónoma de Barcelona. Ejerce como profesor visitante en el Departamento de Comunicación de la Universidad Pompeu Fabra, donde coordina el grupo de investigación CAS. Sus principales líneas de investigación son la publicidad y las relaciones públicas en medios digitales, el branding político y la producción sonora. Ha publicado trabajos sobre contenidos mediáticos en dispositivos inteligentes, valores en la publicidad y calidad comunicativa, géneros y modelos entonativos en el discurso informativo.
\end{abstract}

http://orcid.org/0000-0003-2239-4082

Iluis.mas@upf.edu

Universitat Pompeu Fabra Roc Boronat, 138. 08018 Barcelona, España

\section{Resumen}

Twitter se ha convertido en uno de los principales medios de difusión de mensajes políticos (tweets) compuestos de cuatro elementos - texto, etiquetas o hashtags, menciones y enlaces- que se presentan, ordenan y repiten con la única limitación de un máximo de 140 caracteres. El objetivo de este trabajo es analizar cómo los usuarios de una conversación política estructuran sus tweets, y qué impacto tiene la estructura en la difusión o retweet de sus mensajes. Se analizan dos muestras de más de 500.000 tweets cada una, correspondientes a las elecciones generales españolas de 2015 y 2016 . Los resultados muestran diferencias significativas en el orden y cantidad de elementos de los tweets utilizados por políticos, medios, periodistas y ciudadanos. En particular, los políticos tienden a una mayor utilización de hashtags, y los medios más enlaces. La difusión del tweet depende de la presencia de enlaces, el número de seguidores y el número de hashtags. Este trabajo añade conocimiento al estudio de las estrategias de políticos, medios y periodistas en la elaboración de tweets en campañas electorales.

\section{Palabras clave}

Comunicación política; Twitter; Tweets; Medios sociales; Influencia; Estructura de los tweets; Elecciones; Estrategias comunicativas; España.

\begin{abstract}
Twitter has become one of the main media for spreading political messages. The structure of these messages, or tweets, is compounded by four elements -text, hashtags, mentions, and links- that can be used, organized, and repeated with the only limitation of 140 characters at most. The objective of this work is to analyze how users of political conversations gave structure to their tweets, and what the impact this structure has in the spread or retweet of these messages. We analyze two samples of more than half a million tweets each, related to the Spanish general elections in 2015 and 2016 . The results
\end{abstract}


show significant differences in the order and amount of elements in the tweets used by politicians, media, journalists, and citizens. In particular, politicians tend to make a greater use of hashtags, and media use more links. The spread of tweets depends upon the presence of these links, the number of followers, and the number of hashtags. This work contributes with knowledge of the strategies applied by politicians, media, and journalists in the elaboration of electoral campaign tweets.

\section{Keywords}

Political communication; Twitter; Tweets; Social media; Influence; Structure of a tweet; Elections; Communication strategies; Spain.

Guerrero-Solé, Frederic; Mas-Manchón, Lluís (2017). “Estructura de los tweets políticos durante las campañas electorales de 2015 y 2016 en España”. El profesional de la información, v. 26, n. 5, pp. 805-815.

https://doi.org/10.3145/epi.2017.sep.03

\section{Introducción}

La comunicación política es pionera en el uso de nuevos medios, tecnologías y estrategias comunicativas. Actualmente es un ámbito fuertemente determinado por la personalización de las campañas electorales (Nusselder, 2013), la construcción del candidato como marca (Cwalina; Falkowski, 2015) y la creación de comunidades virtuales (Stieglitz; Brockmann; Xuan, 2012). Aunque se mantienen los formatos en los medios tradicionales, la comunicación política de los partidos está adoptando un modelo basado en una cultura participativa e interactiva (Winther-Nielsen, 2017) en la que el mensaje político en Twitter es un elemento clave.

En pocos años Twitter se ha convertido en uno de los principales canales de difusión de mensajes políticos (Graham; Jackson; Broersma, 2016). En la bibliografía académica se suele situar las elecciones norteamericanas de 2012 como el momento de consolidación de Twitter como espacio de comunicación política (Guerrero-Solé, 2017). A ello ayudó la victoria de Obama, quien lo utilizó de forma profusa en su segunda campaña electoral (Conway; Kenski; Wang, 2015).

Desde 2010 se ha llevado a cabo un gran número de investigaciones sobre el impacto de Twitter en la comunicación política. Muchos de estos trabajos se han centrado en su adopción por parte de políticos, periodistas o celebridades (Bruns; Burgess, 2012; Bruns; Highfield, 2013; Himelboim, 2014; Molyneux, 2015; Graham; Jackson; Broersma, 2016), así como en el análisis de los datos como forma de extracción de información relevante para predecir los resultados electorales. Los análisis de la red (network analysis) han servido para visualizar la estructura de la red de usuarios, o predecir determinados atributos latentes de los mismos. En general se ha llegado a conclusiones parecidas sobre el comportamiento de los usuarios, quienes tienden a interactuar con gente con los mismos intereses (Himelboim; McCreery; Smith, 2013; Huberty, 2015), o sobre la polarización política (Guerrero-Solé, 2017), entre otras.

Algunos de los trabajos pioneros se han centrado en aspectos formales y de contenido, aportando información acerca de la importancia de las imágenes o los enlaces para maximizar el impacto del mensaje en forma de retweets (Liu; Liu; Li, 2012; Zhang et al., 2014; Lahuerta-Otero; Cordero-Gutiérrez, 2016). Sin embargo hay pocos estudios sistemáticos de la forma que pueden adoptar los tweets. Este trabajo analiza la estructura de los tweets políticos durante las dos últimas campañas electorales en España con el objetivo de conocer el tipo de estructuras dominantes utilizadas por los usuarios en este campo y evaluar el impacto que tienen los aspectos formales en la difusión de los mensajes. Estos resultados pueden servir para conocer las estrategias de los partidos políticos y los medios asociadas a la estructura del tweet, así como para formular hipótesis sobre las funciones comunicativas de esta estructura.

\section{La popularización de Twitter ha permiti- do fijar convenciones en la configuración de los cuatro elementos de la estructura de un tweet: hashtags, enlaces, mencio- nes y texto}

\section{Estructura del tweet}

Twitter es considerada una red de microblogging fundamentalmente conversacional (Barash; Golder, 2011). Sus características formales y comunicativas se derivan de la escritura web, en particular de los blogs, los foros o los posts (Menna, 2012), pero se remontan por su brevedad hasta la época del telégrafo o de la cultura oral (Puschmann, 2015; Heyd; Puschmann, 2016). Twitter es un medio de comunicación escrita, aunque la limitación del espacio a 140 caracteres, su inmediatez, horizontalidad y obsolescencia lo acercan al lenguaje oral coloquial (Van-Dijk, 2013).

Más allá de la limitación a los 140 caracteres, Twitter plantea un nuevo nivel comunicativo, que consiste en la ordenación y repetición de 4 tipos de elementos:

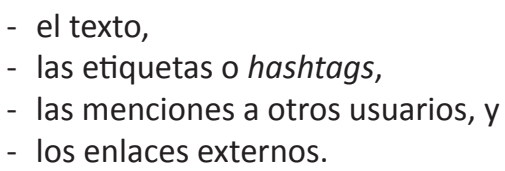

La conjugación de estos cuatro elementos no responde de forma clara a un código preestablecido, sino que es fruto de la decisión de cada usuario. A pesar de la aparente libertad y falta de reglas (Lahuerta-Otero; Cordero-Gutiérrez, 2016), en el decurso de su popularización como red social se han fijado determinadas convenciones que permiten dar estructura a los mensajes: 
- la sintaxis @usuario sirve para mencionar e interpelar a determinados usuarios;

- la \#etiqueta se utiliza para marcar los temas de conversación (hashtags) y facilitar la recuperación de tweets de ese campo temático (Suh et al., 2010).

El tweet puede incorporar además contenidos externos a través de urls (enlaces) (Boyd; Golder; Lotan, 2010).

Algunos trabajos han estudiado la presencia de los enlaces en comunidades de usuarios en torno a un hashtag (Holton et al., 2014; Puschmann, 2015), o el valor significativo del hashtag como tal (Menna, 2012).

A nivel lingüístico, Menna (2012) plantea que el hashtag puede tener un valor pragmático-discursivo y un valor gramatical:

- el hashtag actúa de nodo comunicativo que canaliza un tema sobre el que una comunidad de usuarios debate. Esta función puede estar relacionada con la estructuración de los cuatro elementos por criterios estilísticos o estéticos, pues hay usuarios que utilizan el medio para reconocerse y ser reconocidos como parte de una comunidad de usuarios (Boyd; Golder; Lotan, 2010);

- el hashtag puede tener un valor léxico, es decir, puede actuar como unidad sintáctica y semántica completa o combinarse con el resto de elementos para formar una unidad gramatical. Hasta el momento se han constatado casos en que el hashtag ha actuado como frase subordinada o como sujeto del texto del tweet (Menna, 2012). Además, se puede combinar de forma complementaria o suplementaria con otros hashtags.

Hasta la fecha no existen investigaciones sobre el uso e impacto de las formas que puede adoptar un tweet a partir de la ordenación y repetición de los cuatro elementos. De este modo, nuestras primeras preguntas de investigación son:

PI1a: ¿Cuál es la distribución de las estructuras que puede tener un tweet en una conversación política?

PI1b: ¿Hay diferencias en la distribución de las estructuras más frecuentes en función del tipo de usuarios (políticos, medios, periodistas y ciudadanos) y de las campañas electorales?

PI1c: ¿Cómo influyen los elementos de las estructuras de los tweets y el número de seguidores de los usuarios en la frecuencia en que estas estructuras son utilizadas?

Una de las principales líneas de investigación en Twitter es el análisis de la influencia de los usuarios. Aunque en un principio el número de seguidores se presentó como el factor definitivo de influencia, algunas de las investigaciones hechas hasta el momento señalan otros factores, como la difusión y repercusión de los tweets (Cha et al., 2010). Desde el principio los estudios sobre Twitter han analizado el uso de hashtags y enlaces entre comunidades, y su frecuencia en los tweets y retweets (Letierce et al., 2010) como factor fundamental para una mayor repercusión en la red (Suh et al., 2010). Otros estudios plantean que los tweets más largos tienen mayor influencia social (Lahuerta-Otero; Cordero-Gutiérrez, 2016).

En este sentido cabe destacar que la influencia en Twitter es fruto de un esfuerzo continuado de los usuarios, y no algo ganado espontánea o accidentalmente (Cha et al., 2010). Del mismo modo que los periodistas en los blogs (Singer, 2005), los periodistas en Twitter tienden a normalizar sus prácticas en la red y a adoptar determinadas normas y estándares (Lasorsa; Lewis; Holton, 2012). Además los candidatos políticos utilizan los hashtags en los procesos electorales para crear marcas políticas alrededor de las cuales se configuran comunidades online (Cwalina; Falkowski, 2015). Por la propia naturaleza del medio, la difusión de los mensajes es el principal objetivo de los cuatro tipos de usuarios:

PI2a: ¿Con qué frecuencia utilizan los usuarios los elementos de los que se compone un tweet? ¿Hay diferencias entre tipos de usuarios y entre campañas electorales?

PI2b: ¿Cómo influyen los elementos de un tweet y el número de seguidores de los usuarios en su redifusión (retweet)?

\section{Método y muestra}

Para analizar la estructura de los tweets se desarrolló un método que permite diferenciar los elementos de un tweet, que hemos marcado como:

- menciones a otros usuarios [@],

- hashtags [\#];

- enlaces [L];

- cualquier cadena de texto al principio, final o entre dos elementos [T].

De este modo, para cada tweet se obtiene una cadena con su estructura y se calcula el número de elementos que lo componen, como en el ejemplo siguiente:

Al mensaje:

Hoy @usuario1 atenderá a las preguntas de los \#lectores sobre el \#20N. Accede a este enlace para verlo en directo $h t t p: / / e n l a c e . c o m$

le corresponde la estructura $[T][@][T][\#][T][\#][T][L]$ formada por 8 elementos.

Desde un punto de vista probabilístico, el número de combinaciones viene dado por los 4 tipos de elementos identificados y el límite de caracteres, teniendo en cuenta que no puede haber dos elementos [T] seguidos. Si consideramos además que: el número de [L] se restringe a 3; por término medio, el número de caracteres de un usuario y de un hashtag es de 10; y asumimos un máximo de 12 elementos (el máximo en la muestra es de 29 , y la media de 8,9 ), el número final de combinaciones posibles puede superar las $200.000\left(4^{2 *} 3^{5 *} 2^{5}\right.$ más las combinaciones con menos de 12 elementos hasta llegar a 1 ).

A continuación calculamos la frecuencia de cada una de las estructuras y obtenemos, entre otros datos, el número de veces que los tweets con una determinada estructura fueron retweeteados (RT) y el número medio de seguidores (Fol) de los usuarios que publicaron un tweet con la estructura.

El método se aplicó a dos muestras de más de medio millón de mensajes cada una, recogidas durante las dos últimas campañas de las elecciones generales de España, celebradas el 20 de diciembre de 2015 (20D), y el 26 de junio de 
2016 (26J). Las muestras se componen de tweets políticos difundidos por partidos políticos, medios, periodistas y ciudadanos, y marcados con los hashtags neutros \#20D y \#26J. Para la recogida de los mensajes se utilizó la API Search de Twitter entre el 3 y el 18 de diciembre de 2015, y entre el 9 y el 24 de junio de 2016, respectivamente para cada muestra. Así pues, todos los mensajes analizados tienen en común que como mínimo incluyen uno de los dos hashtags.

A pesar de que la elección de hashtags para recoger una muestra puede condicionar la propia muestra (los mensajes incluían obligatoriamente un hashtag neutro), la segmentación de la muestra a partir del hashtag general de las elecciones representa presumiblemente un espacio de interacción controlado y no sesgado (Cohen; Ruths, 2013). Aparte del sesgo de la inclusión del hashtag genérico debemos añadir los sesgos relativos al proceso de recolección de datos (Tufekci, 2014) y al funcionamiento de los algoritmos de las APIs de Twitter como cajas negras (Bruns, 2012) que afectan a la muestra. En la tabla 1 se muestran las descripciones de ambas muestras.

\section{Aunque el número de combinaciones posibles supera las 200.000 , los cuatro tipos de usuarios utilizan entre 8 y 10 es- tructuras diferentes}

Además de la recogida y análisis de los tweets, clasificamos los usuarios de ambas muestras en cuatro categorías:

- partidos y políticos,

- medios,

- periodistas, y

- ciudadanos.

El proceso de clasificación se realizó en tres fases:

- los 5.000 usuarios más retweeteados (tabla 2);

- aquellos que, ya sea en el nombre de usuario o en la descripción, se identificaban explícitamente como partidos, miembros de partido, medios o periodistas;

- todos aquellos con más de 1.000 seguidores.

De este modo quedaron clasificados los usuarios con más influencia, ya sea por el alcance de la redifusión de sus mensajes, o por el número de seguidores.

\section{Resultados}

Los 110.523 mensajes de la muestra final del \#20D fueron clasificados en 9.741 estructuras diferentes, y los 101.166 del \#26J en 8.968.

La distribución de las estructuras es muy desigual. En ambas muestras, el $1 \%$ de las estructuras incluyen aproximadamente el $60 \%$ de los casos, por lo que, aunque no de forma exacta, la distribución se aproxima a la de una ley de potencia.

En cuanto al número de elementos de los tweets, se sitúa mayoritariamente entre 3 y 8 , con una distribución que se acerca a la normalidad. La distribución de los elementos es diferente según el tipo de usuario. En la tabla 3 vemos las tres estructuras más repetidas para políticos, medios, pe-
Tabla 1. Descripción de las muestras de \#20D y \#26J

\begin{tabular}{|l|c|c|}
\cline { 2 - 3 } \multicolumn{1}{c|}{} & \#20D & \#26J \\
\hline Mensajes recogidos & 540.849 & 576.842 \\
\hline Tweets & $110.523(20,4 \%)$ & $101.166(17,5 \%)$ \\
\hline Retweets nativos & $430.326(79,6 \%)$ & $475.676(82,5 \%)$ \\
\hline
\end{tabular}

Tabla 2. Clasificación de los 5.000 usuarios más retweeteados en \#20D y \#26J

\begin{tabular}{|l|c|c|}
\cline { 2 - 3 } \multicolumn{1}{c|}{} & \#20D & \#26J \\
\hline Políticos & 1.946 & 2.202 \\
\hline Medios & 194 & 182 \\
\hline Periodistas & 225 & 213 \\
\hline Ciudadanos y otros & 2.635 & 2.403 \\
\hline
\end{tabular}

riodistas y ciudadanos durante ambas campañas. El número de elementos de un tweet tiene relación con la frecuencia de ocurrencia de la estructura. A mayor cantidad de texto, hashtags o menciones, menor frecuencia de ocurrencia. Este resultado se explica en parte por la baja probabilidad de ocurrencia de tweets con gran número de elementos. Sin embargo, los datos de la tabla III del anexo nos aportan información añadida respecto a qué estructuras son utilizadas más allá del número de elementos. El número de tweets que empiezan con un enlace es casi menospreciable, y el de los que empiezan por una mención está por debajo del $8 \%$ en todos los casos. De este modo, observamos que una gran mayoría de los mensajes (sobre el 75\%) empiezan por texto, y entre el $15 \%$ y el $25 \%$ con un hashtag. Este listado se complementa con las tablas I y II del anexo.

Tabla 3. Listado de las 3 estructuras más frecuentes entre políticos, medios, periodistas y ciudadanos en 20D y 26J.

\begin{tabular}{|c|c|c|c|}
\hline \multicolumn{2}{|r|}{ 20D } & \multicolumn{2}{|c|}{ 26J } \\
\hline Frec. & Estructura & Estructura & Frec. \\
\hline \multicolumn{4}{|c|}{ Políticos } \\
\hline 853 & {$[\mathrm{~T}][\#][\#][\mathrm{L}]$} & {$[\mathrm{T}][\#][\#][\mathrm{L}]$} & 1.248 \\
\hline 832 & {$[\mathrm{~T}][\#][\mathrm{T}][\#][\mathrm{L}]$} & {$[\mathrm{T}][\#][\mathrm{T}][\#][\mathrm{L}]$} & 1.092 \\
\hline 637 & {$[\mathrm{~T}][\#][\#][\#][\mathrm{L}]$} & {$[\mathrm{T}][\#][\#][\#][\mathrm{L}]$} & 769 \\
\hline \multicolumn{4}{|c|}{ Medios } \\
\hline 304 & {$[\mathrm{~T}][\#][\mathrm{L}][\mathrm{L}]$} & {$[\mathrm{T}][\#][\mathrm{L}][\mathrm{L}]$} & 406 \\
\hline 279 & {$[\mathrm{~T}][\#][\mathrm{L}]$} & {$[\mathrm{T}][\#][\mathrm{L}]$} & 295 \\
\hline 264 & {$[\mathrm{~T}][\mathrm{L}][\#][\mathrm{L}]$} & {$[\mathrm{T}][\#][\mathrm{T}][\mathrm{L}][\mathrm{L}]$} & 238 \\
\hline \multicolumn{4}{|c|}{ Periodistas } \\
\hline 413 & {$[\mathrm{~T}][\#][\mathrm{L}]$} & {$[\mathrm{T}][\#][\mathrm{L}]$} & 335 \\
\hline 377 & {$[\mathrm{~T}][\#]$} & {$[\mathrm{T}][\#]$} & 233 \\
\hline 161 & {$[\mathrm{~T}][\#][\mathrm{T}]$} & {$[\mathrm{T}][@][\mathrm{T}][\#][\mathrm{L}]$} & 121 \\
\hline \multicolumn{4}{|c|}{ Ciudadanos } \\
\hline 3.393 & {$[\mathrm{~T}][\#][\mathrm{L}]$} & {$[\mathrm{T}][\#][\mathrm{L}]$} & 2.630 \\
\hline 3.195 & {$[\mathrm{~T}][\#]$} & {$[\mathrm{T}][\#]$} & 2.080 \\
\hline 2.857 & {$[\mathrm{~T}][\#][\mathrm{T}]$} & {$[\mathrm{T}][\#][\mathrm{T}][\mathrm{L}]$} & 1.848 \\
\hline
\end{tabular}


Para resolver la PI1c calculamos las posibles correlaciones entre el número de veces que se repite una determinada estructura y los elementos que contiene, así como la correlación con las veces que son retweeteados los mensajes con esta estructura, y el número medio de seguidores de los usuarios que tweetearon mensajes con la estructura. En la tabla 4 se muestran las correlaciones en el caso de los políticos y los medios en ambas campañas.

Además de las correlaciones, se propone un modelo de regresión lineal múltiple para determinar el número de veces que una estructura se repetía en función de los elementos de la estructura y los seguidores medios de los usuarios que la utilizaban. Para los usuarios políticos, las cuatro variables predecían de forma estadísticamente significativa el valor de S_Num, $F(5,3588)=1.077 .418, p<0,001, R^{2}=0,775$ para 20D. Ocurría lo mismo para 26J: $F(5,3576)=1.592 .166, p<$ $0,001, R^{2}=0,690$.

Los resultados fueron muy parecidos para los medios, con:

- $F(5,1103)=741.663, p<0,001, R^{2}=0,771$, en 20D

- $F(5,1033)=351.970, p<0,001, R^{2}=0,630$, en 26J

Como puede verse en la tabla 5 , la variable número de seguidores prácticamente no contribuye a explicar la frecuencia de la estructura. El resto de variables contribuyen significativamente con coeficientes negativos, excepto el número de enlaces.

\section{Los políticos utilizan estructuras con 2 ó} más hashtags y los medios tienden a incluir enlaces

En el caso de los periodistas, las regresiones dieron como resultado:

- $F(5,1137)=43,952, p<0,001, R^{2}=0,162$, en $20 D$

- $F(5,1006)=299,434, p<0,001, R^{2}=0,598$, en 26J

En el de los ciudadanos:

- $F(5,6638)=702,829, p<0,001, R^{2}=0,346$, en 20D

- $F(5,5678)=119,814, p<0,001, R^{2}=0,095$, en 26J

Aunque los resultados son estadísticamente significativos, el número de casos explicados $\left(R^{2}\right)$ es mucho menor que en políticos y medios.

Tabla 5. Valores de beta y significación estadística de las regresiones múltiples lineales para políticos y medios en 20D y $26 \mathrm{~J}$.

\begin{tabular}{|l|c|c|c|c|}
\cline { 2 - 5 } \multicolumn{1}{c|}{} & \multicolumn{2}{c|}{ Políticos } & \multicolumn{2}{c|}{ Medios } \\
\cline { 2 - 5 } \multicolumn{1}{c|}{} & 20D & 26J & 20D & 26J \\
\hline (Constante) & $16,850^{* * *}$ & $14,960^{* * *}$ & $11,643^{* * *}$ & $12,297^{* * *}$ \\
\hline Fol & $1,738 \mathrm{E}-5^{* * *}$ & $1,334 \mathrm{E}-5^{* * *}$ & $2,828 \mathrm{E}-6^{* * *}$ & $3,329 \mathrm{E}-6^{* * *}$ \\
\hline$[\#]$ & $-1,421^{* * *}$ & $-1,287^{* * *}$ & $-1,617^{* * *}$ & $-1,526^{* * *}$ \\
\hline$[\mathrm{L}]$ & $-1,665^{* *}$ & $-0,422$ & $-0,734$ & $-1,281$ \\
\hline$[\mathrm{T}]$ & $-1,359^{* * *}$ & $-1,321^{* * *}$ & $-0,718^{*}$ & $-0,948^{*}$ \\
\hline$[@]$ & $-2,270^{* * *}$ & $-2,051^{* * *}$ & $-1,541^{* * *}$ & $-1,513^{* * *}$ \\
\hline
\end{tabular}

$* \mathrm{p}<0,05 * * \mathrm{p}<0,01 * * * \mathrm{p}<0,001$
Tabla 4. Correlaciones entre el número de veces que se repite una estructura y los elementos que la componen, el número de elementos (EI), los retweets recibidos (RT) y el número medio de seguidores (Fol).

\begin{tabular}{|l|c|c|c|c|}
\cline { 2 - 5 } \multicolumn{1}{c|}{} & \multicolumn{2}{c|}{ Políticos } & \multicolumn{2}{c|}{ Medios } \\
\cline { 2 - 5 } & 20D & 26J & 20D & 26J \\
\hline RT & $0,664^{* *}$ & $0,796^{* *}$ & $0,895^{* *}$ & $0,797^{* *}$ \\
\hline Fol & $0,768^{* *}$ & $0,828^{* *}$ & $0,871^{* *}$ & $0,787^{* *}$ \\
\hline$[\#]$ & $-0,080^{* *}$ & $-0,078^{* *}$ & $-0,132^{* *}$ & $-0,147^{* *}$ \\
\hline [L] & $-0,017$ & $-0,004$ & $0,064^{*}$ & 0,054 \\
\hline$[T]$ & $-0,072^{* *}$ & $-0,066^{* *}$ & $-0,150^{* *}$ & $-0,154^{* *}$ \\
\hline$[@]$ & $-0,150^{* *}$ & $-0,134^{* *}$ & $-0,185^{* *}$ & $-0,189^{* *}$ \\
\hline El & $-0,216^{* *}$ & $-0,195^{* *}$ & $-0,266^{* *}$ & $-0,269^{* *}$ \\
\hline
\end{tabular}

$* \mathrm{p}<0,05 * * \mathrm{p}<0,01 * * * \mathrm{p}<0,001$

Los coeficientes de la regresión son similares a los de políticos y medios. El número de seguidores no contribuye a la explicación de la variable, pero en este caso la contribución negativa de los enlaces es estadísticamente significativa en todos los casos (tabla 6).

Los partidos políticos pueden decidir aumentar o disminuir el número de determinados elementos del tweet para obtener un determinado impacto

Para responder a la pregunta $\mathrm{PI} 2 \mathrm{a}$ sobre el uso de los elementos de un tweet entre los usuarios, analizamos todos los tweets de ambas campañas y calculamos las medias para cada elemento en 20D y en 26J, el número medio de elementos (EI), la longitud de los tweets (Len), la media de retweets (RT) y seguidores (Fol). Además, para observar su evolución entre las dos campañas, comprobamos mediante una Anova la significación de las diferencias (tabla 7).

Para resolver la última pregunta calculamos primero las correlaciones entre el número de veces que un tweet era retweeteado con el número de elementos y de seguidores.

Tabla 6. Valores de beta y significación estadística de las regresiones múltiples lineales para periodistas y ciudadanos en 20D y 26J

\begin{tabular}{|l|c|c|c|c|}
\cline { 2 - 5 } & \multicolumn{2}{c|}{ Periodistas } & \multicolumn{2}{c|}{ Ciudadanos } \\
\cline { 2 - 5 } & 20D & 26J & 20D & 26J \\
\hline (Constante) & $24,879^{* * *}$ & $12,505^{* * *}$ & $70,946^{* * *}$ & $75,371^{* * *}$ \\
\hline Fol & $1,921 \mathrm{E}-6^{* * *}$ & $1,257 \mathrm{E}-5^{* * *}$ & $1,997 \mathrm{E}-5^{* * *}$ & $3,190 \mathrm{E}-6^{* * *}$ \\
\hline$[\#]$ & $-2,917^{* * *}$ & $-1,144^{* * *}$ & $-6,957^{* * *}$ & $-7,707^{* * *}$ \\
\hline$[\mathrm{L}]$ & $-4,376^{* * *}$ & $-2,102^{* * *}$ & $-12,205^{* * *}$ & $-10,808^{* * *}$ \\
\hline$[\mathrm{T}]$ & $-1,482^{* * * *}$ & $-1,112^{* * *}$ & $-5,097^{* * *}$ & $-5,482^{* * *}$ \\
\hline$[@]$ & $-3,558^{* * *}$ & $-1,923^{* * *}$ & $-8,814^{* * *}$ & $-9,512^{* * *}$ \\
\hline
\end{tabular}

$* \mathrm{p}<0,05 * * \mathrm{p}<0,01 * * * \mathrm{p}<0,001$ 
Tabla 7. Medias del número de elementos de los tweets, su longitud (Len), número de retweets recibidos (RT) y media del número de seguidores (Fol).

\begin{tabular}{|l|c|c|c|c|c|c|c|c|}
\hline & [\#] & [L] & [T] & [@] & [EI] & Len & RT & Fol \\
\hline \multicolumn{7}{|c|}{ Políticos } \\
\hline 20D & 2,61 & 0,79 & 2,07 & 0,83 & 6,30 & 119,80 & 10,93 & 9.907 \\
\hline 26J & 2,52 & 0,83 & 2,10 & 0,85 & 6,29 & 120,78 & 11,76 & 16.793 \\
\hline Dif & $-0,09^{* *}$ & $0,04^{* *}$ & $0,03^{* *}$ & $0,02^{*}$ & $-0,01$ & $0,98^{* *}$ & $0,83^{* *}$ & $6.886^{* *}$ \\
\hline \multicolumn{7}{|c|}{ Medios } \\
\hline 20D & 1,55 & 1,36 & 1,91 & 0,74 & 5,56 & 121,07 & 7,56 & 142.545 \\
\hline 26J & 1,56 & 1,27 & 1,84 & 0,76 & 5,43 & 122,03 & 4,91 & 137.284 \\
\hline Dif & 0,01 & $-0,09^{* *}$ & $-0,07^{* *}$ & 0,02 & $-0,13^{* *}$ & $0,96^{* *}$ & $-2,26^{* *}$ & -5.261 \\
\hline
\end{tabular}

\begin{tabular}{|l|c|c|c|c|c|c|c|c|}
\hline \multicolumn{1}{|c|}{ Periodistas } \\
\hline 20D & 1,76 & 0,74 & 1,97 & 0,78 & 5,25 & 117,13 & 5,31 & 5.405 \\
\hline 26J & 1,81 & 0,75 & 1,99 & 0,91 & 5,46 & 116,33 & 4,14 & 8.854 \\
\hline Dif & $0,05^{*}$ & 0,01 & 0,02 & $0,13^{* *}$ & $0,21^{* *}$ & $-0,80$ & $-1,17^{*}$ & $3.449^{* *}$ \\
\hline
\end{tabular}

\begin{tabular}{|l|c|c|c|c|c|c|c|c|}
\hline \multicolumn{7}{|c|}{ Ciudadanos } \\
\hline 20D & 2,18 & 0,71 & 1,91 & 0,63 & 5,43 & 112,60 & 6,22 & 2.951 \\
\hline 26J & 2,26 & 0,74 & 1,93 & 0,72 & 5,66 & 114,65 & 6,06 & 3.761 \\
\hline Dif & $0,08^{* *}$ & $0,03^{* *}$ & $0,02^{* *}$ & $0,09 * *$ & $0,23^{* *}$ & $2,05^{* *}$ & $-0,16$ & $810^{* *}$ \\
\hline
\end{tabular}

$* p<0,05 * * p<0,01 * * * p<0,001$

Aunque no sean rasgos estructurales, el número de seguidores está altamente correlacionado con el número de retweets y el número de enlaces tiene una contribución positiva. En cambio, el resto de variables tienden a correlacionarse negativamente de forma desigual.

Los resultados de las regresiones múltiples nos muestran que el modelo predictivo, a pesar de ser estadísticamente significativo, explica un número relativamente pequeño de casos. Para los políticos, los resultados son :

- $F(5,25759)=1370,714, p<0,001, R^{2}=0,210$, en $20 D$

- $F(5,30200)=1507,997, p<0,001, R^{2}=0,200$, en 26J

Para los medios:

- $F(5,6487)=100.152, p<0,001, R^{2}=0,072$, en $20 D$

- $F(5,6326)=207.878, p<0,001, R^{2}=0,141$, en $26 \mathrm{~J}$

Tabla 8. Correlaciones entre el número de retweets y los elementos que componen el tweet (EI) y el número de seguidores (Fol), para políticos y medios.

\begin{tabular}{|l|c|c|c|c|}
\cline { 2 - 5 } \multicolumn{1}{|c|}{} & \multicolumn{2}{c|}{ Políticos } & \multicolumn{2}{c|}{ Medios } \\
\cline { 2 - 5 } & $\mathbf{2 0 D}$ & $\mathbf{2 6 J}$ & $\mathbf{2 0 D}$ & $\mathbf{2 6 J}$ \\
\hline Fol & $0,453^{* *}$ & $0,441^{* *}$ & $0,237^{* *}$ & $0,372^{* *}$ \\
\hline$[\#]$ & $-0,021^{* *}$ & 0,007 & 0,008 & $-0,027^{*}$ \\
\hline$[\mathrm{L}]$ & $0,037^{* *}$ & 0,011 & $0,134^{* *}$ & $0,040^{* *}$ \\
\hline$[\mathrm{T}]$ & $0,016^{*}$ & $-0,024^{* *}$ & $-0,036^{* *}$ & 0,010 \\
\hline$[@]$ & $-0,021$ & $-0,039^{* *}$ & $-0,091^{* *}$ & 0,012 \\
\hline El & $-0,007$ & $-0,026^{* *}$ & $-0,017$ & 0,014 \\
\hline
\end{tabular}

$* \mathrm{p}<0,05 * * \mathrm{p}<0,01 * * * \mathrm{p}<0,001$
Los coeficientes de la regresión y su significación se muestran en la tabla 9.

Los resultados para los periodistas y ciudadanos no son concluyentes y muestran comportamientos opuestos entre las dos elecciones.

\section{Conclusiones}

Los resultados de este trabajo contribuyen al conocimiento de las estrategias de los usuarios en la estructuración de los tweets en una conversación política definida por un hashtag neutro. Además, el análisis de las dos elecciones consecutivas en España en 2015 y 2016 nos ha permitido ver la evolución de estas estrategias en el tiempo.

Por un lado, el estudio demuestra que el número de estructuras diferentes es limitado, y que los usuarios sólo utilizan de forma sistemática un número reducido de éstas. Así, a pesar de que el número de combinaciones de elementos posibles es muy grande, se observa una uniformización de las estructuras que demuestra la existencia de una cierta normatividad.

Por otro lado, el uso de estas estructuras dominantes depende del tipo de usuario. En general, los políticos tienden a utilizar estructuras con dos o más hashtags, en consonancia con los resultados de investigaciones anteriores (Alonso-Muñoz; Miquel-Segarra; Casero-Ripollés, 2016), y los medios tienden a incorporar enlaces en sus tweets. Se puede plantear la hipótesis de que los políticos añaden marcas propias a los tweets y los medios incorporan enlaces a sus noticias.

Los enlaces son la única variable formal que contribuye positiva y significativamente a la redifusión de los tweets

A pesar de que Twitter ha sido concebida como una red

Tabla 9. Valores de beta y significación estadística de las regresiones múltiples lineales para periodistas y ciudadanos en 20D y 26J.

\begin{tabular}{|l|c|c|c|c|}
\cline { 2 - 5 } \multicolumn{1}{c|}{} & \multicolumn{2}{c|}{ Políticos } & \multicolumn{2}{c|}{ Medios } \\
\cline { 2 - 5 } & 20D & 26J & 20D & 26J \\
\hline (Constante) & 0,054 & $5,730^{* * *}$ & 1,557 & $2,071^{* * *}$ \\
\hline$[\mathrm{H}]$ & $0,827^{* * *}$ & $1,020^{* * *}$ & $0,472^{* * *}$ & 0,238 \\
\hline$[\mathrm{L}]$ & $5,350^{* * *}$ & $3,376^{* * *}$ & $3,206^{* * *}$ & 0,458 \\
\hline$[\mathrm{T}]$ & 0,008 & $-1,165^{* * *}$ & 0,141 & $-0,510$ \\
\hline$[@]$ & $-0,530^{*}$ & $-0,596^{* * *}$ & $-0,797^{* * *}$ & $1,162^{* * *}$ \\
\hline Fol & $0,000^{* * *}$ & $0,000^{* * *}$ & $8,669 \mathrm{E}-6^{* * *}$ & $1,422 \mathrm{E}-5^{* * *}$ \\
\hline
\end{tabular}

$* \mathrm{p}<0,0 * * \mathrm{p}<0,01 * * * \mathrm{p}<0,001$ 
conversacional, la mención es en general el elemento menos utilizado. Sólo el $8 \%$ de los mensajes empiezan con una mención, y la media de menciones está muy por debajo de 1 en todos los casos analizados. Esta conclusión coincide con la de estudios anteriores que demuestran que los partidos políticos no aprovechan el potencial dialógico de Twitter y apuestan por la unidireccionalidad, con independencia de su ideología o número de seguidores (Alonso-Muñoz; Miquel-Segarra; Casero-Ripollés, 2016; Ribalko; Seltzer, 2010). Sin embargo, la desmiente parcialmente, ya que nuestros resultados muestran que los medios, periodistas y ciudadanos utilizan menos menciones que los partidos políticos. Este resultado es relevante y debería poner en cuestión el supuesto desaprovechamiento del potencial dialógico de Twitter por parte de los políticos.

\section{Los medios, periodistas y ciudadanos utilizan menos menciones que los parti- dos políticos}

En cuanto al impacto de los tweets en función de los elementos que contiene, este trabajo refuerza la importancia de los enlaces en la redifusión de los mensajes. Los enlaces son la única variable formal que contribuye positiva y significativamente a la redifusión de los mensajes, como ya se ha demostrado en estudios anteriores (Liu; Liu; Li, 2012; Lahuerta-Otero; Cordero-Gutiérrez, 2016). El modelo de regresión aplicado a los políticos y los medios muestra que el número de hashtags contribuye mínimamente a aumentar la redifusión, mientras que las menciones contribuyen a reducirla. Este resultado llevaría a la conclusión de que el uso de Twitter como herramienta dialógica, que conlleva la inclusión de menciones a otros usuarios, no es una estrategia que mejore la redifusión del mensaje. Y si la redifusión es el objetivo, estos resultados pueden explicar su escaso uso. Sin embargo, deberíamos examinar con mayor detalle las diferencias entre no incluir menciones o incluir una o dos para detallar su influencia en la redifusión. A pesar de ello, el número de retweets puede no ser el único criterio para incluir o no menciones, ya que su influencia está también en incrementar el diálogo entre políticos y ciudadanía, por ejemplo. Más allá de la estructura del tweet, el análisis también concluye que el número de seguidores influye significativamente y está altamente correlacionado con el número de retweets, pero contribuye débilmente a su explicación en la regresión lineal múltiple.

Estos resultados aportan conocimiento a la comunicación política en Twitter. Por una parte, se trata de un análisis de las estrategias políticas y las estrategias de medios y periodistas durante las campañas electorales. Los partidos políticos pueden decidir aumentar o disminuir el número de determinados elementos para obtener un determinado impacto en la red. También es útil como base para la armonización de los mensajes de los partidos políticos, que son publicados desde centenares de cuentas y que, en muchas ocasiones, tienen formas muy diferentes y confusas. Asimis- mo, estos resultados dan a conocer las rutinas de medios y periodistas para crear un tweet político.

Por otra parte, el estudio plantea aprender la sintaxis de la red social en función del tipo de usuario y facilitar el proceso de ajuste y adaptación al medio por parte de los nuevos usuarios o de aquellos que quieren optimizar sus formas de expresión. En este sentido, la creación de mensajes políticos en las redes depende de la educación y de la intensidad de uso (Casero-Ripollés, 2017), por lo que estos factores también pueden influir en las estructuras de los mensajes de los ciudadanos.

No obstante, la gramática del tweet es necesariamente incipiente por cuanto este hipotético lenguaje no se ha institucionalizado a través de un uso colectivo sistemático. Como nivel significativo potencialmente comunicativo, el rango de criterios que pueden determinar las diferentes configuraciones de los cuatro elementos en un tweet es muy amplio, y constituye un objeto de investigación que abarca diferentes tipos de usuarios, hashtags, culturas o contextos.

Futuros estudios deberán observar todo tipo de criterios para articular los cuatro elementos: semánticos, semióticos, estilísticos y estéticos.

Además, la estructura del tweet podría ser objeto de estudio de la teoría del discurso con modelos basados en la estructuración temática de los elementos de una estructura para una transmisión optimizada de la información, y que han sido aplicados en campos que alcanzan la lingüística computacional y la generación de lenguaje natural.

Puede ser de interés para políticos y medios conocer cuáles son las combinaciones que maximizan el impacto de sus mensajes en la red y adaptar sus rutinas a ello

Este trabajo tiene algunas limitaciones, entre las que destacan la selección de la muestra con tweets que contienen un hashtag, y los ya citados sesgos de la recolección de datos con las APIs de Twitter (Tufekci, 2014; Bruns, 2012) que pueden tener un impacto en la generalización de los resultados y su significación. En trabajos posteriores deberemos comprobar cuáles son las estructuras más repetidas sin este tipo de restricciones. Además, el trabajo es insuficiente para comprobar cómo el uso de estructuras varía en el tiempo. Aunque las dos elecciones españolas de 2015 y 2016 son un buen ejemplo para comprobar la dinámica de las estructuras e hipotetizar algunos de los cambios que se producen entre ambas, será necesario investigar futuras conversaciones para observar si hay patrones que se mantienen en el tiempo y si el aprendizaje y las estrategias privilegian de forma recurrente determinadas formas. Asumiendo que un mismo mensaje puede ser reproducido con estructuras diferentes, puede ser de interés para políticos y medios conocer cuáles son las combinaciones que maximizan el impacto de sus mensajes en la red y adaptar sus rutinas a ello. 


\section{Bibliografía}

Alonso-Muñoz, Laura; Miquel-Segarra, Susana; Casero-Ripollés, Andreu (2016). "Un potencial comunicativo desaprovechado. Twitter como mecanismo generador de diálogo en campaña electoral". Obra digital, v. 11, pp. 39-59. http://revistesdigitals.uvic.cat/index.php/obradigital/ article/view/100/106

Barash, Vladimir; Golder, Scott (2011). "Twitter: Conversation, entertainment, and information, all in one network!". En: Hansen, Derek L.; Shneiderman, Ben; Zhang, Marc A. (eds). Analyzing social media networks with NodeXL, pp. 143-164. ISBN: 9780123822291

https://doi.org/10.1016/B978-0-12-382229-1.00010-2

Boyd, Danah; Golder, Scott; Lotan, Gilad (2010). "Tweet, tweet, retweet: Conversational aspects of retweeting on Twitter". En: Procs of the Annual Hawaii intl conf on system sciences.

https://doi.org/10.1109/HICSS.2010.412

Bruns, Axel (2012). "How long is a tweet? Mapping dynamic conversation networks on Twitter using Gawk and Gephi". Information, communication \& society, v. 15, n. 9, pp. 13231351.

https://eprints.qut.edu.au/47819/

https://doi.org/10.1080/1369118X.2011.635214

Bruns, Axel; Burgess, Jean (2012). "Researching news discussion on Twitter. New metodologies". Journalism studies, v. 13 , n. 5-6, pp. 801-14.

http://snurb.info/files/2012/Researching\%20News\%20 Discussion\%20on\%20Twitter.pdf

https://doi.org/10.1080/1461670X.2012.664428

Bruns, Axel; Highfield, Tim (2013). "Political networks on Twitter. Tweeting the Queensland state election". Information, communication \& society, v. 16, n. 5, pp. 667-691.

http://snurb.info/files/2013/Political\%20Networks\%20 on\%20Twitter.pdf

https://doi.org/10.1080/1369118X.2013.782328

Casero-Ripollés, Andreu (2017). “Producing political content for web 2.0: Empowering citizens and vulnerable populations". El profesional de la información, v. 26, n. 1, pp. 13-19. https://doi.org/10.3145/epi.2017.ene.02

Cha, Meeyoung; Haddadi, Hamed; Benevenuto, Fabrício; Gummadi, Krishna P. (2010). "Measuring user influence in Twitter: The million follower fallacy". En: Procs of the $4^{\text {th }}$ Intl AAAl conf on weblogs and social media, Icwsm 2010, pp. 10-17.

http://twitter.mpi-sws.org/icwsm2010_fallacy.pdf

Cohen, Raviv; Ruths, Derek (2013). “Classifying political orientation on Twitter: It's not easy!". In: $7^{\text {th }}$ Intl AAAl conf on weblogs and social media, pp. 91-99.

https://www.aaai.org/ocs/index.php/ICWSM/ICWSM13/ paper/viewFile/6128/6347

Conway, Bethany A.; Kenski, Kate; Wang, Di (2015). “The rise of Twitter in the political campaign: Searching for intermedia agenda-setting effects in the presidential primary". Journal of computer-mediated communication, v. 20, n. 4, pp. 363-380. https://doi.org/10.1111/jcc4.12124

Cwalina, Wojciech; Falkowski, Andrzej (2015). “Political branding: Political candidates positioning based on inter-object associative affinity index". Journal of political marketing, v. 14, n. 1-2, pp. 152-174.

https://doi.org/10.1080/15377857.2014.990842

Graham, Todd; Jackson, Dan; Broersma, Marcel (2016). "New platform, old habits? Candidates' use of Twitter during the 2010 British and Dutch general election campaigns". New media \& society, v. 18, n. 5, pp. 765-783.

http://eprints.whiterose.ac.uk/113492/

https://doi.org/10.1177/1461444814546728

Guerrero-Solé, Frederic (2017). “Community detection in political discussions on Twitter: An application of the retweet overlap network method to the Catalan process toward independence". Social science computer review, n. 35, v. 2.

https://goo.gl/2F75Kq

https://doi.org/10.1177/0894439315617254

Heyd, Theresa; Puschmann, Cornelius (2016). “Hashtagging and functional shift: Adaptation and appropriation of the \#". Journal of pragmatics, v. 116, pp. 51-63.

https://goo.gl/ytznpv

https://doi.org/10.1016/j.pragma.2016.12.004

Himelboim, Itai (2014). "Political television hosts on Twitter: Examining patterns of interconnectivity and self-exposure in Twitter political talk networks". Journal of broadcasting \& electronic media, v. 58, n. 1, pp. 76-96.

https://doi.org/10.1080/08838151.2013.875017

Himelboim, Itai; McCreery, Stephen; Smith, Marc (2013). "Birds of a feather tweet together: Integrating network and content analyses to examine cross-ideology exposure on Twitter". Journal of computer-mediated communication, $\mathrm{v}$. 18 , n. 2, pp. 40-60.

https://doi.org/10.1111/jcc4.12001

Holton, Avery E.; Baek, Kang; Coddington, Mark; Yaschur, Carolyn (2014). "Seeking and sharing: Motivations for linking on Twitter". Communication research reports, v. 31, n. 1, pp. 33-40.

https://goo.gl/Unw1nZ

https://doi.org/10.1080/08824096.2013.843165

Huberty, Mark (2015). "Can we vote with our tweet? On the perennial difficulty of election forecasting with social media". International journal of forecasting, v. 31, n. 3, pp. 992-1007.

https://goo.gl/pkuLbZ

https://doi.org/10.1016/j.ijforecast.2014.08.005

Lahuerta-Otero, Eva; Cordero-Gutiérrez, Rebeca (2016). "Looking for the perfect tweet. The use of data mining techniques to find influencers on Twitter". Computers in human behavior, v. 64, pp. 575-583.

https://doi.org/10.1016/j.chb.2016.07.035

Lasorsa, Dominic L.; Lewis, Seth C.; Holton, Avery E. (2012). "Normalizing Twitter. Journalism practice in an emerging communication space". Journalism studies, v. 13, n. 1, pp. 19-36. 
https://apps.cla.umn.edu/directory/items/publication/316405. $p d f$

https://doi.org/10.1080/1461670X.2011.571825

Letierce, Julie; Passant, Alexandre; Decker, Stefan; Breslin, John G. (2010). "Understanding how Twitter is used to spread scientific messages". En: Procs of the WebSci10. Extending the frontiers of society on-line, April 26-27, Raleigh, NC, US. http://journal.webscience.org/314

Liu, Zhiming; Liu, Lu; Li, Hong (2012). "Determinants of information retweeting in microblogging". Internet research, v. 22 , n. 4 , pp. $443-66$.

https://goo.gl/trZwkB

https://doi.org/10.1108/10662241211250980

Menna, Laura (2012). "Nuevas formas de significación en red: el uso de las \#etiquetas en el movimiento 15M". Estudios de lingüística del español (ELiEs), 34.

http://elies.rediris.es/elies34/Tesina_L-Menna.pdf

Molyneux, Logan (2015). "What journalists retweet: Opinion, humor, and brand development on Twitter". Journalism, v. 16, n. 7, pp. 920-935.

https://doi.org/10.1177/1464884914550135

Nusselder, André (2013). "Twitter and the personalization of politics". Psychoanalysis, culture \& society, v. 18, n. 1, pp. 91-100.

https://goo.gl/fJKXKg

https://doi.org/10.1057/pcs.2012.45

Puschmann, Cornelius (2015). "The form and function of quoting in digital media". Discourse, context and media, v. 7, pp. 28-36.

https://goo.gl/VsuYgD

https://doi.org/10.1016/j.dcm.2015.01.001

Ribalko, Svetlana; Seltzer, Trent (2010). “Dialogic communication in 140 characters or less: How Fortune 500 companies engage stakeholders using Twitter". Public relations review, v. 36, n. 4, pp. 336-341.

https://goo.gl/ixvKum https://doi.org/10.1016/j.pubrev.2010.08.004

Singer, Jane B. (2005). "'The political J-blogger: 'normalizing' a new media form to fit old norms and practices'". Journalism, v. 6, n. 2, pp. 173-198.

http://openaccess.city.ac.uk/3463/

https://doi.org/10.1177/1464884905051009

Stieglitz, Stefan; Brockmann, Tobias; Xuan, Linh-Dang (2012). "Usage of social media for political communication". In: Pacis 2012 proceedings, paper 22.

http://aisel.aisnet.org/pacis2012/22

Suh, Bongwon; Hong, Lichan; Pirolli, Peter; Chi, Ed H. (2010). "Want to be retweeted? Large scale analytics on factors impacting retweet in Twitter network". In: Procs SocialCom 2010: $2^{\text {nd }}$ IEEE Int conf on social computing. Passat 2010: $2^{\text {nd }}$ IEEE Intl conf on privacy, security, risk and trust, Minneapolis, Minnesota, USA, August 20-22, pp. 177-184. https://goo.gl/1EoVvW https://doi.org/10.1109/SocialCom.2010.33

Tufekci, Zeinep (2014). "Big questions for social media big data: Representativeness, validity and other methodological pitfalls". En: Icwsm'14: Procs of the $8^{\text {th }}$ Intl AAAl Conf on weblogs and social media, pp. 505-514.

https://arxiv.org/abs/1403.7400

Van-Dijck, José (2013). The culture of connectivity. A critical history of social media. New York, NY: Oxford University Press. ISBN: 9780199970780

Winther-Nielsen, Sige (2017). “On political brands: A systematic review of the literature". Journal of political marketing, v. 16, n. 2, pp. 118-146.

https://doi.org/10.1080/15377857.2014.959694

Zhang, Lun; Peng, Tai-Quan; Zhang, Ya-Peng; Wang, XiaoHong; Zhu, Jonathan H. (2014). "Content or context: Which matters more in information processing on microblogging sites". Computers in human behavior, v. 31, n. 1, pp. 242-49. https://goo.gl/NEimbN https://doi.org/10.1016/j.chb.2013.10.031 


\section{Anexos}

Tabla I. Estructuras de tweets más frecuentes entre políticos y medios en 20D de 2015 y 26J de 2016

\begin{tabular}{|c|c|c|c|c|}
\hline $\mathbf{N}$ & Frec & Estructura & Estructura & Frec. \\
\hline \multicolumn{5}{|c|}{ Políticos } \\
\hline & \multicolumn{2}{|r|}{ 20D } & \multicolumn{2}{|l|}{ 26J } \\
\hline 1 & 853 & {$[\mathrm{~T}][\#][\#][\mathrm{L}]$} & {$[\mathrm{T}][\#][\#][\mathrm{L}]$} & 1.248 \\
\hline 2 & 832 & {$[\mathrm{~T}][\#][\mathrm{T}][\#][\mathrm{L}]$} & {$[\mathrm{T}][\#][\mathrm{T}][\#][\mathrm{L}]$} & 1.092 \\
\hline 3 & 637 & {$[\mathrm{~T}][\#][\#][\#][\mathrm{L}]$} & {$[T][\#][\#][\#][L]$} & 769 \\
\hline 4 & 518 & {$[\mathrm{~T}][\#][\mathrm{T}][\mathrm{L}]$} & {$[\mathrm{T}][\#][\mathrm{T}][\mathrm{L}]$} & 636 \\
\hline 5 & 399 & {$[\mathrm{~T}][\#]$} & {$[T][\#][T][\#][\#][L]$} & 621 \\
\hline 6 & 389 & {$[\mathrm{~T}][\#][\mathrm{L}]$} & {$[\mathrm{T}][@][\mathrm{T}][\#][\#][\mathrm{L}]$} & 588 \\
\hline 7 & 384 & {$[\mathrm{~T}][\#][\mathrm{T}][\#][\#][\mathrm{L}]$} & {$[\mathrm{T}][\#][\mathrm{L}]$} & 574 \\
\hline 8 & 351 & {$[T][@][T][\#][T][\#]$} & {$[T][\#][T][\#][T][L]$} & 428 \\
\hline 9 & 299 & {$[\mathrm{~T}][\#][\#]$} & {$[\mathrm{T}][\#][T][@][\#]$} & 420 \\
\hline 10 & 297 & {$[\mathrm{~T}][@][\mathrm{T}][\#][\#][\mathrm{L}]$} & {$[\mathrm{T}][@][\mathrm{T}][\#][\mathrm{T}][\#][\mathrm{L}]$} & 364 \\
\hline 11 & 296 & {$[T][\#][T][\#][T][L]$} & {$[\mathrm{T}][\#][\mathrm{T}][\#][\mathrm{T}][\#][\mathrm{L}]$} & 356 \\
\hline 12 & 295 & {$[\mathrm{~T}][@][\mathrm{T}][\#][\mathrm{T}][\#][\mathrm{L}]$} & {$[T][\#][T][@][\#][L]$} & 299 \\
\hline 13 & 288 & {$[\mathrm{~T}][\#][\#][\#][\#][\mathrm{L}]$} & {$[\#][\#][\#][\#][L]$} & 280 \\
\hline 14 & 249 & {$[\mathrm{~T}][\#][\mathrm{T}][\#]$} & {$[T][@][T][\#][\#]$} & 276 \\
\hline 15 & 242 & {$[\mathrm{~T}][\#][\mathrm{T}][\#][\mathrm{T}][\#][\mathrm{L}]$} & {$[T][@][T][\#][\#][\#][L]$} & 275 \\
\hline
\end{tabular}

\begin{tabular}{|l|r|l|l|r|}
\hline \multicolumn{5}{|c|}{ Medios } \\
\hline 1 & 304 & {$[T][\#][L][L]$} & {$[T][\#][L][L]$} & 406 \\
\hline 2 & 279 & {$[T][\#][L]$} & {$[T][\#][L]$} & 295 \\
\hline 3 & 264 & {$[T][L][\#][L]$} & {$[T][\#][T][L][L]$} & 238 \\
\hline 4 & 197 & {$[\#][T][L][L]$} & {$[\#][T][L]$} & 226 \\
\hline 5 & 193 & {$[T][\#][T][L][L]$} & {$[\#][T][L][L]$} & 214 \\
\hline 6 & 181 & {$[T][L][\#]$} & {$[T][L][\#][L]$} & 197 \\
\hline 7 & 180 & {$[T][\#][T][L]$} & {$[T][@][T][\#][L]$} & 158 \\
\hline 8 & 166 & {$[\#][T][L]$} & {$[T][\#][T][L]$} & 129 \\
\hline 9 & 103 & {$[T][\#][T][\#][L][L]$} & {$[T][L][\#]$} & 128 \\
\hline 10 & 98 & {$[T][\#][\#][L][L]$} & {$[T][\#][\#][L]$} & 124 \\
\hline 11 & 91 & {$[T][@][T][L][\#][L]$} & {$[T][L][\#][\#]$} & 93 \\
\hline 12 & 88 & {$[T][@][T][\#][L][L]$} & {$[T][@][T][\#][L][L]$} & 82 \\
\hline 13 & 82 & {$[T][@][T][\#][L]$} & {$[T][@][T][@][T][\#][L]$} & 74 \\
\hline 14 & 80 & {$[T][\#][\#][L]$} & {$[T][\#][T][\#][L]$} & 69 \\
\hline 15 & 74 & {$[\#][\#][T][L][L]$} & {$[T][L][\#][\#][L]$} & 67 \\
\hline
\end{tabular}

Tabla II. Estructuras de tweets más frecuentes entre periodistas y ciudadanos en 20D de 2015 y 26J de 2016

\begin{tabular}{|c|c|c|c|c|}
\hline $\mathbf{N}$ & Frec & Estructura & Estructura & Frec. \\
\hline \multicolumn{5}{|c|}{ Periodistas } \\
\hline & \multicolumn{2}{|r|}{ 20D } & \multicolumn{2}{|l|}{ 26J } \\
\hline 1 & 413 & {$[\mathrm{~T}][\#][\mathrm{L}]$} & {$[\mathrm{T}][\#][\mathrm{L}]$} & 335 \\
\hline 2 & 377 & {$[\mathrm{~T}][\#]$} & {$[\mathrm{T}][\#]$} & 233 \\
\hline 3 & 161 & {$[\mathrm{~T}][\#][\mathrm{T}]$} & {$[T][@][T][\#][L]$} & 121 \\
\hline 4 & 152 & {$[T][@][T][\#][L]$} & {$[\mathrm{T}][\#][\#][\mathrm{L}]$} & 114 \\
\hline 5 & 152 & {$[\mathrm{~T}][\#][\#][\mathrm{L}]$} & [T][L][T][@][@][@][\#][\#] & 111 \\
\hline 6 & 148 & {$[\mathrm{~T}][\#][\mathrm{T}][\mathrm{L}]$} & {$[\mathrm{T}][\#][\mathrm{T}][\mathrm{L}]$} & 111 \\
\hline 7 & 126 & {$[\mathrm{~T}][\#][\#]$} & {$[\mathrm{T}][\#][\#]$} & 111 \\
\hline 8 & 111 & {$[\mathrm{~T}][\#][\mathrm{T}][\#]$} & {$[\mathrm{T}][\#][\mathrm{T}]$} & 101 \\
\hline 9 & 97 & {$[\mathrm{~T}][\#][\mathrm{T}][\#][\mathrm{L}]$} & {$[T][@][T][\#]$} & 100 \\
\hline 10 & 94 & {$[@][\#][T][L]$} & {$[\mathrm{T}][\#][\mathrm{T}][\#][\mathrm{L}]$} & 91 \\
\hline 11 & 75 & {$[T][@][T][\#]$} & {$[\mathrm{T}][\#][\mathrm{T}][\#]$} & 80 \\
\hline 12 & 73 & {$[\mathrm{~T}][\mathrm{L}][\#]$} & {$[\#][\#][\#][T][L]$} & 70 \\
\hline 13 & 61 & {$[T][@][T][\#][T][L]$} & {$[T][\#][L][T][@]$} & 57 \\
\hline 14 & 61 & {$[\mathrm{~T}][\#][\mathrm{T}][@][\mathrm{L}]$} & {$[T][@][T][@][T][\#][L]$} & 54 \\
\hline 15 & 55 & {$[T][L][\#][\#][T][L]$} & {$[\#][T][L][T][@]$} & 53 \\
\hline
\end{tabular}

\begin{tabular}{|l|r|l|l|r|}
\hline \multicolumn{5}{|c|}{ Ciudadanos } \\
\hline \multicolumn{1}{|c|}{} & \multicolumn{5}{|c|}{ 26J } \\
\hline 1 & 3.393 & {$[T][\#][L]$} & {$[T][\#][L]$} & 2.630 \\
\hline 2 & 3.195 & {$[T][\#]$} & {$[T][\#]$} & 2.080 \\
\hline 3 & 2.857 & {$[T][\#][T]$} & {$[T][\#][T][L]$} & 1.848 \\
\hline 4 & 2.800 & {$[T][\#][T][L]$} & {$[T][\#][\#][L]$} & 1.830 \\
\hline 5 & 1.921 & {$[T][\#][\#][L]$} & {$[T][L][T][@][@][@][\#][\#]$} & 1.672 \\
\hline 6 & 1.684 & {$[T][\#][\#]$} & {$[T][\#][T]$} & 1.439 \\
\hline 7 & 1.356 & {$[T][\#][T][\#][L]$} & {$[T][\#][T][\#][L]$} & 1.266 \\
\hline 8 & 1.310 & {$[T][\#][T][\#]$} & {$[T][\#][\#][\#[\#][L]$} & 1.124 \\
\hline 9 & 994 & {$[T][\#][\#][\#][L]$} & {$[T][\#][\#]$} & 1.021 \\
\hline 10 & 987 & {$[\#][T][L]$} & {$[T][\#][T][\#]$} & 1.001 \\
\hline 11 & 817 & {$[T][\#][T][\#][T][L]$} & {$[T][\#][\#][\#][L]$} & 897 \\
\hline 12 & 703 & {$[T][\#][T][\#][T]$} & {$[T][\#][T][\#][T][L]$} & 656 \\
\hline 13 & 648 & {$[T][\#][\#][\#]$} & {$[\#][T][L]$} & 617 \\
\hline 14 & 644 & {$[@][T][\#][T]$} & {$[T][\#][T][\#][T]$} & 540 \\
\hline 15 & 533 & {$[\#][\#][L]$} & {$[T][\#][T][\#][T][\#][T][\#][T]$} & 518 \\
\hline
\end{tabular}


Tabla III. Frecuencia de uso de combinaciones de los tres primeros elementos de un tweet

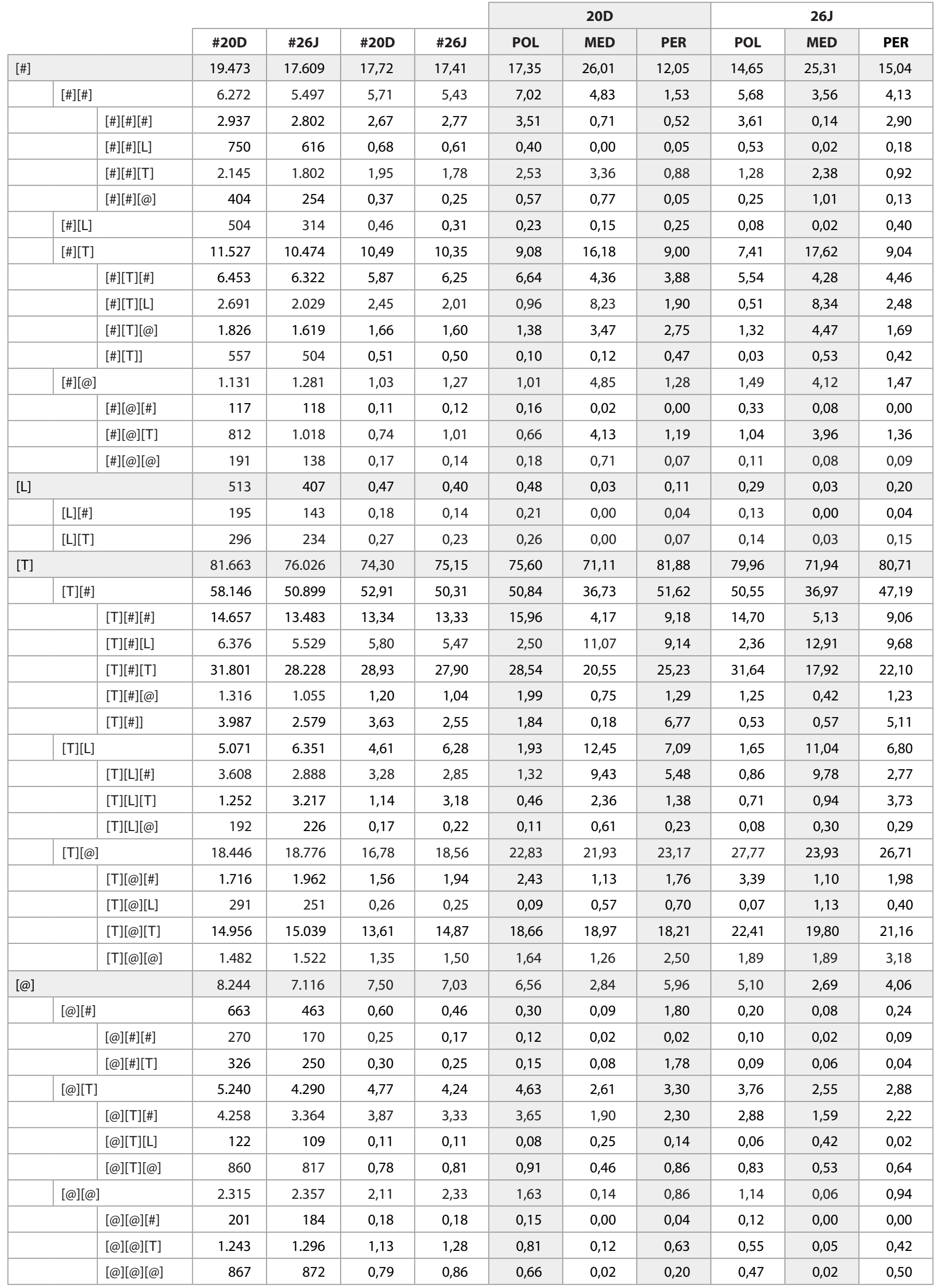

